\title{
The Complex Picture Test in Dementia
}

\author{
K. Gustaw Rothenberga, ${ }^{a} \quad$ R. Piądłod $\quad$ U.D. Nagaraj ${ }^{b}$ \\ R.P. Friedland ${ }^{c}$
}

${ }^{a}$ Department of Psychiatry, University Hospitals, Case Medical Center, Cleveland, Ohio, ${ }^{b}$ Department of Radiology, Ohio State Medical Center, Columbus, Ohio, and

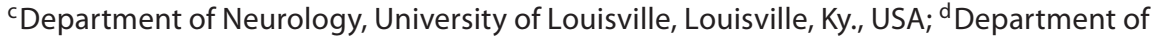
Neurodegenerative Diseases, IMW, Lublin, Poland

\section{Key Words}

Dementia $\cdot$ Cognitive testing $\cdot$ Complex picture test

\begin{abstract}
The newly developed Complex Picture Test (CPT) examines main cognitive domains, especially perceptual and visual-spatial abilities. The potential usefulness of the CPT in the assessment of dementia was evaluated. Patients with different forms of dementia [14 vascular dementia (VaD), 30 Alzheimer's disease (AD), 6 Parkinson's disease dementia (PDD)], 12 subjects with mild cognitive impairment $(\mathrm{MCl})$, and 30 matched controls were examined by the CPT, Mini-Mental State Examination (MMSE), the WAIS-R Digit Span subtest, and the Clock Drawing Test (CDT). The CPT consists of 10 complex pictures, each presenting characteristics paradoxically in opposition to each other. The subjects scored points for naming the object and noticing the paradox. One point was added for commenting on the paradox without prompting ( 3 points each picture/ maximum of 30). CPT scores were significantly higher in the control group (mean \pm SD, 29.46 \pm 1.43 ) than in the $\mathrm{AD}, \mathrm{VaD}, \mathrm{MCl}$, and PDD groups $(13.1 \pm 2.3,16.9 \pm 3.5,23.2 \pm 1.4$, and 23.6 \pm 3.4 , respectively; $p<0.05$ ). Significant positive correlations between MMSE, Digit Span, CDT, and CPT scores were observed (rho $0.76,0.35$, and 0.56 , respectively). These data show perception of complex pictures being compromised in dementia. The correlation between CPT scores and MMSE scores suggests that tests may be used as a brief screening tool for dementia.
\end{abstract}




\section{Introduction}

Alzheimer's disease $(\mathrm{AD})$ is an irreversible, progressive disorder with loss of cognitive function, particularly memory, judgment, reasoning, and perception. The disease primarily affects parts of the association cortex and deep structures including the hippocampus and basal frontal nuclei through a number of pathophysiologic processes. Visual-processing abnormalities commonly contribute to AD symptoms [1]. Studies on visual perception are valuable for understanding the disease and shedding light on what the $\mathrm{AD}$ patients understand. Visual perceptual tests may also help to develop new screening tools for AD. The commonly used neuropsychological tests for dementia, such as the Mini-Mental State Examination (MMSE) or the Montreal Cognitive Assessment, do not evaluate visual-perceptual abilities in context judgment and information processing. The objective of this study was to evaluate the potential utility of a Complex Picture Test (CPT) to assess the existence of the associations between MMSE and CPT and the degree of the decline in dementia. The CPT we have developed involves images presented to the patients with a paradox they had not previously experienced. This presents challenges to the capacity of the subjects to interpret a novel or a complex situation and explain it verbally.

\section{Methods}

\section{Subjects}

The current study is part of a large population-based study, which was designed to assess the prevalence of different forms of dementia in a given population. The participants of the study were randomly selected from the population-based sample within the Lublin region of Poland composed of 2,182,191 inhabitants in the year 2005 when the population was sampled. As a result of the project, the prevalence of $\mathrm{AD}$ in the Lublin area was calculated to be $1,634.6 / 100,000$ inhabitants. The BERCAL study design is described in more detail elsewhere [2]. Controls were selected randomly from the same population.

Patients with a previously established diagnosis of mild cognitive impairment (MCI) or different forms of dementia took part in the study. They were examined in the Department of Neurodegenerative Diseases, Institute of Agricultural Medicine, Lublin, Poland.

The study group was comprised of 14 patients with vascular dementia (VaD), 30 with $\mathrm{AD}, 6$ patients with dementia in Parkinson's disease (PDD), and 12 patients with MCI. The diagnoses were consistent with the Diagnostic and Statistical Manual of Mental Disorders, 4th edition (DSM-IV) criteria and National Institute of Neurologic and Communicative Disorders and Stroke-AD and Related Disorders Association (NINCDS-ADRDA) criteria for possible or probable AD. The Modified Hachinski Ischemic Score [3] was determined; all the patients with $\mathrm{VaD}$ had a composite score of 5 or more and a neuroimaging study consistent with VaD. CT or MRI scans were obtained routinely for all patients and were consistent with the diagnoses. MCI was diagnosed in consistency with Petersen's criteria [4].

Patients included into the study had to be otherwise healthy and ambulatory, with vision and hearing sufficient for compliance with testing procedures. Laboratory test values had to be within normal limits or considered to be clinically insignificant by the physician. All patients had to have a reliable caregiver. Patients were excluded if they had evidence of clinically significant gastrointestinal, renal, hepatic, endocrine, or other systemic disease, epilepsy, primary psychiatric diseases (notably DSM-IV-defined depression), traumatic brain injury, newly treated hypothyroidism, or a known or suspected history (within the past 10 years) of alcoholism or drug abuse. Additional reasons for exclusion included evidence of neoplasm, insulin-dependent diabetes or diabetes not stabilized by diet or oral hypoglycemic 
Table 1. Sociodemographic characteristics of patients and controls

\begin{tabular}{llllll}
\hline Characteristics & $\mathrm{MCI}$ & Control & $\mathrm{VaD}$ & $\mathrm{AD}$ & $\mathrm{PDD}$ \\
\hline $\begin{array}{l}\text { Mean age } \pm \text { SD } \\
\quad \text { (range) }\end{array}$ & $\begin{array}{l}61.3 \pm 10.0 \\
(59-72)\end{array}$ & $\begin{array}{l}64.4 \pm 10.1 \\
(59-84)\end{array}$ & $71.0 \pm 10.9$ & $66.4 \pm 12.1$ & $60.1 \pm 2.2$ \\
$\begin{array}{l}\text { Gender, } \mathrm{n}(\%) \\
\quad \text { Men }\end{array}$ & $5(42)$ & $13(43)$ & $5(42)$ & $17(43.3)$ & $5(83.3)$ \\
$\quad$ Women & $7(58)$ & $17(57)$ & $9(58)$ & $13(56.6)$ & $1(16.6)$ \\
$\begin{array}{l}\text { Residence, } \mathrm{n}(\%) \\
\quad \text { Urban }\end{array}$ & $6(50)$ & $12(40)$ & $2(48)$ & $12(40)$ & $2(33.3)$ \\
$\quad$ Rural & $6(50)$ & $18(60)$ & $12(52)$ & $18(60)$ & $4(66.6)$ \\
\hline
\end{tabular}

Table 2. Description of complex pictures

\begin{tabular}{ll}
\hline Picture No. & Description \\
\hline 1 & The number 4 composed of capital letter B's \\
2 & The number 120 composed of people in a field \\
3 & Rabbit with the coat of a tiger \\
4 & Cow with the map of the world as a pattern on its coat \\
5 & Two people wearing one hat \\
6 & Woman trying to eat spaghetti with an enormous fork \\
7 & Dalmatian dog with multi-colored spots \\
9 & Man with the head of a lion \\
10 & Dog smoking a cigarette \\
\hline
\end{tabular}

agents, obstructive pulmonary disease or asthma, recent ( $<2$ years) hematologic/oncologic disorders, pernicious anemia, or vitamin B12 or folate deficiency. The control group consisted of 30 healthy subjects whose scores on the MMSE were in the range of 28-30. Controls were matched by age and gender and were subject to the same exclusions as the case group (tables 1, 2).

BERCAL was carried out in accordance with the Institute of Agricultural Medicine Bioethical Commission (IRB) and after the protocol was evaluated and approved. After complete description of the study protocol, written informed consent was obtained from the patients (if possible), the caregivers, or the patient's representative (if applicable) before beginning detailed screening activities.

\section{Procedures}

A test composed of 10 complex pictures was administered to patients and controls. The pictures used in the study were of varying complexity, with each consisting of two prominent characteristics that contradicted each other (table 3). All patients were able to see clearly, with vision from 20/20 to 20/40 with or without correction, and took the test with their best corrected vision in a well-lit room. Each picture was shown to the patients who were asked to describe it and to comment on the picture. The picture was given to the patients, and they were able to hold it to view it in whatever position they preferred. Points were scored for naming the object in the picture, noticing the paradox, and commenting on the paradox appropriately after seeing the picture. Naming the object in the picture and noticing the paradox reflected our test points and were scored visual-spatial abilities, and commenting on the 
Table 3. Clinical characteristics of the patients with dementia as measured by MMSE and CDT (mean \pm $\mathrm{SD})$

\begin{tabular}{lrcrrr}
\hline Test & \multicolumn{1}{l}{ MCI } & Control & \multicolumn{1}{l}{ VaD } & \multicolumn{1}{l}{ AD } & \multicolumn{1}{l}{ PDD } \\
\hline MMSE & $25.7 \pm 1.1$ & $28.7 \pm 1.4$ & $21.3 \pm 7.2$ & $14.3 \pm 6.1$ & $23.3 \pm 2.4$ \\
CDT free drawing & $8.5 \pm 2.3$ & 10 & $5.3 \pm 2.0$ & $3.2 \pm 4.1$ & $8.8 \pm 1.2$ \\
CDT copying & $9.2 \pm 1.6$ & 10 & $7.3 \pm 2.0$ & $3.0 \pm 3.7$ & $9.2 \pm 1.6$ \\
Digit Span & $5.3 \pm 1.7$ & $5.8 \pm 1.5$ & $4.5 \pm 1.3$ & $4.3 \pm 2.0$ & $5.8 \pm 1.9$
\end{tabular}

Table 4. Performance on CPT in examined groups of patients compared using one-way analysis of variance

\begin{tabular}{llllll}
\hline Test & Controls & MCI & AD & VaD & PDD \\
\hline Mean CDT score \pm SD & $29.5 \pm 1.4$ & $23.6 \pm 1.4^{*}$ & $13.1 \pm 2.3^{*}$ & $16.9 \pm 3.5^{*}$ & $23.3 \pm 2.4^{*}$ \\
\hline
\end{tabular}

* All pair-wise multiple comparison achieved a significance level of $\mathrm{p}<0.05$ as compared with controls.

paradox appropriately reflected insight and judgment. Thus, the subjects could score 3 points for each picture, with a maximum of 30 points altogether.

The patients also received the MMSE [5, 6] and the Clock Drawing Test (CDT) following the procedure suggested by Shah [7] and the scoring system developed by Manos [8] as they combine simplicity, quantitative scoring, and further qualitative information relevant for clinical diagnosis. Each patient was first given a sheet of paper with a pre-drawn circle signifying the clock face and asked to complete it with appropriate numbers and clock hands indicating 11:10 (the free drawing version). Then, the patient was given a complete, predrawn picture of the clock and instructed to copy it (the copying version). The free drawing version is assumed to examine language functions (verbal comprehension of the command), memory functions (recalling visual engrams stored in the long-term memory and recalling the instruction on the time on which the clock hands were to be set/stored in the short-term memory), and executive functions (planning, sequencing, organizing), which generally correspond to the temporal and frontal lobe activities. The copying version is less dependent on language and memory function as it activates mainly perceptual and visual-spatial abilities associated with the parietal lobe control. The scoring system involved the evaluation of the spatial correctness of the numbers written in the clock face and the appropriateness of the clock hands showing the indicated time. One point was given for any of the numbers 1, 2, 4, $5,7,8,10$, and 11 if they were placed in the correct area of the clock face. One point was also given for the short hand indicating number 11, and for the long hand indicating number 2 . The possible score range is from 0 to 10 , with higher scores indicating better performance. Scores lower than 8 indicate an increased likelihood of intellectual impairment, and scores lower than 5 evidence severe intellectual impairment $[8,9]$.

Additionally, the WAIS-R Digit Span subtest, which examines attention and short term memory (the scores range from $0-8$ ), was implemented. Patients were asked to remember a series of 8 digits and repeat them to the examiner. Each digit repeated in a correct order scored 1 point. 


\section{Statistical Analysis}

The results are presented as means \pm standard deviations (SD). The differences between the examined groups and controls were tested by ANOVA. The associations between the scores on the CPT and the scores on the other tests were calculated as Spearman's rho correlation coefficients. The results of the statistical tests were considered significant at a level of $\mathrm{p}<0.05$.

\section{Results}

The CPT scores were significantly higher in the control group $(29.5 \pm 1.4)$ than in the $\mathrm{AD}, \mathrm{VaD}, \mathrm{MCI}$, and PDD groups $(13.1 \pm 2.3,16.9 \pm 3.5,23.2 \pm 1.4$, and $23.6 \pm 3.4$, respectively; $\mathrm{p}<0.05)$ (table 4).

Significant positive correlations between MMSE, Digit Span, and CDT scores and CPT scores were observed (rho $0.76,0.35$, and 0.56 , respectively).

\section{Discussion}

This study proposes a new measure for the assessment of visual-perceptual deficits in $\mathrm{AD}$ patients. The CPT significantly correlates with the MMSE, the most widely used measurement for the assessment of the severity in AD [10]. Based on clinical experience, it can be generalized that the greater the severity of $\mathrm{AD}$, the deeper the impairment on this visual perception measure. The results of the analysis of variance indicate that particular items on this new scale significantly differentiate between $\mathrm{AD}$ patients and controls.

There are many reasons why visual perceptions of complex pictures are compromised in AD patients. Turner's [11] study suggests that contrast sensitivity plays a significant role in the ability of AD patients to see pictures clearly. Even though all the subjects were tested for visual acuity, such testing is performed under high-contrast settings and thus does not take into account differences in contrast sensitivity. It has been reported that increasing the stimulus contrast significantly increases cognitive performance in AD patients [12]. Visual perception of complex pictures is compromised in $\mathrm{AD}$ because of the impaired function of the parietal-occipital cortex.

Furthermore, visual-special recognition impairment in patients with AD usually accompanies but also impairment of judgment and information processing. This set of dysfunctional abilities may influence many different aspects of everyday life and may create danger in certain situation such as, for example, driving and operating complicated tools or machinery.

The most common example of a potentially dangerous situation where the person relies on both the visual-spatial component of cognition and clear judgment would be driving. There are data showing that driving crashes in patients with $\mathrm{AD}$ should be recognized as an important public health problem [13].

Bringing in mind the above-mentioned data, it seems not surprising that the risk of car accidents is higher in patients with $\mathrm{AD}$ [14]. The risk is attributable not only to visual impairment but also to slowed mentation and attention. Since these attention mechanisms are impaired in $\mathrm{AD}$, the $\mathrm{AD}$ patient's ability to interpret a picture with two distracting features is compromised. Patients who cannot properly scan the visual field and perceive a number made up by smaller parts (table 3) may be unfit for driving as driving requires a large effective field of vision [14]. 
The tool which will closely address impaired visual functioning, judgment, and information processing may be potentially used to assess the ability of a patient to be involved in activities of a certain level of exposure to danger like the one involved in driving. Although the CPT has not been correlated with driving ability or with accidents, it may, however, be considered as a candidate for a tool in the assessment of safe driving abilities.

Another neurologic defect in $\mathrm{AD}$ that could impair the ability to interpret complex pictures is the presence of functional disconnectivity. Neocortical disconnectivity is the inability to integrate sensory information processed in separate cortical regions [15]. Neurodegeneration of the retina itself is also found in $\mathrm{AD}$, with structural damage of the optic nerve head and death of retinal ganglion cells [16]. The nerve fiber layer in the retina of AD patients is reduced, which correlates with abnormal electroretinogram recordings, demonstrating retinal dysfunction [17]. These data suggest that visual perception is significantly impaired in patients with dementia. The strong correlation between the CPT scores and MMSE scores suggests that tests of visual perception could be used as a brief screening tool for dementia.

The mental status examination is often a stressful experience for the patient and family, and the CPT is valuable for providing temporary relief. The images are shared with the family after they are seen by the patient, and the humor in many images (such as the tiger rabbit, table 3) often lightens the seriousness of the moment. It is also valuable for the family to see the extent of the patient's impairment as denial of illness is often shared by the patients and their families and can interfere with compliance with advice concerning care and hazardous behaviors [18].

The aim of our study was to turn clinical, observation based phenomenon which is impaired complex pictures understanding and interpretation in demented patients to more structured, valid test. The proposed tool requires, however, more extensive study in terms of population tested, statistics implemented, and comparison with existing screening as well as diagnostic tools. We plan to examine a larger sample of people with dementia using the CPT tool.

\section{Acknowledgements}

Supported in part by AG017173, the University of Louisville Foundation, and The Mason and Mary Rudd Family.

\section{Disclosure Statement}

The authors have no conflicts of interest to disclose.

\section{References}

-1 Mentis MJ, Horwitz B, Grady CL, Alexander GE, VanMeter JW, Maisog JM, Pietrini P, Schapiro MB, Rapoport SI: Visual cortical dysfunction in Alzheimer's disease evaluated with a temporally graded 'stress test' during PET. Am J Psychiatry 1996;153:32-40.

2 Gustaw K, Woznica I, Bylina J: Rozpowszechnienie zespolow otepiennych w tym choroby Alzheimera w populacji mieszkancow wojewodztwa lubelskiego. Medycyna Ogolna 2008;4:381-394.

-3 Rosen WG, Terry RD, Fuld PA, Katzman R, Peck A: Pathological verification of ischemic score in differentiation of dementias. Ann Neurol 1980;7:486-488.

-4 Petersen RC, Smith GE, Waring SC, Ivnik RJ, Tangalos EG, Kokmen E: Mild cognitive impairment: clinical characterization and outcome. Arch Neurol 1999;56:303. 
-5 Crum RM, Anthony JC, Basset SS, Folstein FM: Population-based norms for the Mini-Mental State Examination by age and educational level. JAMA 1993;269:2386-2391.

-6 Folstein FM, Robins LN, Helzer JE: The Mini-Mental State Examination. Arch Gen Psychiatry 1983; 40:812.

7 Shah J: Only time will tell: clock drawing as an early indication of neurological dysfunction. P\&S Med Rev 2001;7:30-34.

8 Manos PJ: The utility of the ten-point clock test as a screen for cognitive impairment in general hospital patients. Gen Hosp Psychiatry 1997;19:439-444.

-9 Libson DJ, Swenson RA, Barnoski EJ, Sands LP: Clock drawing as an assessment tool for dementia. Arch Clin Neuropsychol 1993;8:405-415.

-10 Mendiondo MS, Ashford JW, Kryscio RJ, Schmitt FA: Modelling mini mental state examination changes in Alzheimer's disease. Stat Med 2000;19:1607-1616.

11 Turner J: Visual perception in normal aging and Alzheimer's disease: influences on picture naming and recognition. OhioLINK ETD 1990.

-12 Gilmore GC, Cronin-Golomb A, Neargarder SA, Morrison SR: Enhanced stimulus contrast normalizes visual processing of rapidly presented letters in Alzheimer's disease. Vision Res 2005;45:10131020.

-13 Friedland RP, Koss E, Kumar A, Gaine S, Metzler D, Haxby JV, Moore A: Motor vehicle crashes in dementia of the Alzheimer type. Ann Neurol 1988;24:782-786.

-14 Dawson JD, Anderson SW, Uc EY, Dastrup E, Rizzo M: Predictors of driving safety in early Alzheimer disease. Neurology 2009;72:521-527.

-15 Festa EK, Insler RZ, Salmon DP, Paxton J, Hamilton JM, Heindel WC: Neocortical disconnectivity disrupts sensory integration in Alzheimer's disease. Neuropsychology 2005;19:728-738.

16 Osborne NN, Schmidt KG: Neuroprotection against glaucoma remains a concept (in German). Ophthalmologe 2004;101:1087-1092.

17 Parisi V: Correlation between morphological and functional retinal impairment in patients affected by ocular hypertension, glaucoma, demyelinating optic neuritis and Alzheimer's disease. Semin Ophthalmol 2003;2:50-57.

18 Weinstein EA, Friedland RP, Wagner EE: Denial/unawareness of impairment and symbolic behavior in Alzheimer’s disease. Neuropsychiatry Neuropsychol Behav Neurol 1994;7:176-184. 